\title{
WIIBRAGEC
}

\section{ANÁLISE DAS PERIODICIDADES DE MANUTENÇÃO PREDIAL NOS SISTEMAS ELÉTRICO E HIDROSSANITÁRIO ${ }^{1}$}

\section{GROLLI, Jhonatan (1); TOSIN, Carlos Eduardo (2); ADUATI, Jean Lucas (3); COSTELLA, Marcelo Fabiano (4)}

Universidade Comunitária da Região de Chapecó, jhonatan.grolli@unochapeco.edu.br (2) Universidade Comunitária da Região de Chapecó, carlos.tosin@udesc.br, (3) Faculdade Meridional, jeanlucasaduati@hotmail.com, (4) Universidade Comunitária da Região de Chapecó, costella@unochapeco.edu.br

\begin{abstract}
RESUMO
O objetivo foi realizar uma comparação entre as periodicidades de manutenção propostas pelo Guia da CBIC e as normas e documentos técnicos disponiveis no âmbito nacional. Os dados para comparação foram extraidos de normas técnicas e fabricantes de forma online, foram analisadas as normas pertinentes para os sistemas, manuais de instalação, manuais técnicos, manuais de uso, informações técnicas $e$ o certificado dos produtos, buscando localizar informações a respeito da periodicidade da manutenção predial para o sistema hidrossanitário e elétrico. Posteriormente foi realizado um comparativo entre os dados obtidos e o guia nacional para elaboração do manual de uso realizado pela Câmara Brasileira da Indústria da Construção - CBIC. A revisão documental resultou em 23 normas do sistema hidrossanitário e 22 do elétrico analisadas, entre elas apenas a NBR 5674 possui informações a respeito da periodicidade da manutenção. Para os fabricantes foram analisadas 87 empresas e não foram encontradas informações sobre o assunto que pudessem contribuir para o comparativo. A análise comparativa entre os dados demonstrou que as atividades e periodicidades propostas não são similares às informações técnicas coletadas. Conclui-se que as informações que constam no guia da CBIC não estão disponíveis em normas técnicas nacionais e nem pelos fabricantes.
\end{abstract}

Palavras-chave: Manutenção Predial, Periodicidade de manutenção, Durabilidade, Instalações.

\begin{abstract}
The objective was to compare the maintenance frequency proposed by the CBIC Guide and the standards and technical documents available at the national level. The data for comparison were extracted from technical standards and manufacturers online. The relevant standards for the systems, installation manuals, technical manuals, user manuals, technical information, and the product certificate were analyzed, seeking to find information about the frequency from building maintenance to the hydro-sanitary and electrical system. Subsequently, a comparison was made between the data obtained and the national guidelines for preparing the user manual carried out by the Brazilian Chamber of Construction Industry CBIC. The document review resulted in 23 standards for the hydro-sanitary system, and 22 for the electric system analyzed, among which only NBR 5674 has information on the periodicity of maintenance. For manufacturers, 87 companies were analyzed, and no information on the subject that could contribute to the comparison was found. The comparative analysis between the data showed that the activities and maintenance frequencies proposed are not similar to the technical information collected. It is concluded that the information contained in the CBIC guide is not available in national technical standards or by manufacturers.
\end{abstract}

Keywords: Building Maintenance, Maintenance Frequency, Durability, Facilities.

I GROLLI, J.; TOSIN, C. E.; ADUATI, J. L.; COSTELLA, M. F. Análise das periodicidades de manutenção predial nos sistemas elétrico e hidrossanitário. In: SIMPÓSIO BRASILEIRO DE GESTÃO E ECONOMIA DA CONSTRUÇÃO, 12., 2021, Maceió, Alagoas. Anais[...] Porto Alegre: ANTAC, 2021. p.1-8. Disponível em: https://eventos.antac.org.br/index.php/sibragec/article/view/513. Acesso em: 2 out. 2021. 


\section{INTRODUÇÃO}

A construção civil ganha cada vez mais espaço no mercado brasileiro, sendo responsável por uma grande fração da economia. Desta forma, os investimentos voltados à mão de obra e à tecnologia, com o intuito de aperfeiçoar e otimizar os processos de construção, são vultosos (CBIC, 2018). Nessa área é comum a preocupação com qualidade e produtividade. Contudo, a manutenção predial das edificações não está entre as prioridades dos usuários durante a fase de utilização, mesmo que ações preventivas representem grande importância e influenciem diretamente na qualidade, conforto e segurança dos usuários da edificação (MAURÍCIO, 2011).

Existe uma necessidade de mudança cultural em relação à manutenção predial, pois ainda é encarada como custo pelos usuários da edificação (VIEIRA, 2015), além disso, muitas vezes é realizada de maneira indevida, em desacordo com as normas e com os manuais do usuário e das áreas comuns. Fruto disso, é que falhas e anomalias decorrentes dos sistemas elétrico, hidrossanitário, climatização, mecânico e proteção contra incêndio são as que afetam de forma mais crítica aos usuários e o desempenho da edificação (DAS; CHEW, 2011; BORTOLINI; FORCADA, 2018).

Existem normas com a finalidade de orientar na manutenção das edificações: NBR 5674 (ABNT, 2012), que fixa os procedimentos de orientação para organização de um sistema de manutenção de edificações, e a NBR 14037 (ABNT, 2014), que aborda as operações de um edifício e procedimentos recomendáveis para a manutenção. Outras normas associadas são a NBR 15575 (ABNT, 2013), voltada ao desempenho de edificações habitacionais, e a NBR 16280 (ABNT, 2015), voltada às reformas em edificações.

Mesmo diante dessas normas, uma barreira é a carência de informação a respeito da periodicidade da manutenção predial. Por vezes, a periodicidade é estabelecida pelas construtoras/incorporadoras, pela empresa que fornece o produto e, na maioria dos casos, adota-se as periodicidades informadas no Guia Nacional para a Elaboração do Manual de Uso, Operação e Manutenção das Edificações (CBIC, 2014). Entretanto, é duvidosa a fonte de informações utilizada para informar a periodicidade das manutenções preventivas, uma vez que este assunto é pouco relatado nas normas brasileiras.

Assim, o objetivo foi realizar uma comparação entre as periodicidades propostas pelo Guia da CBIC, para a manutenção do sistema elétrico e hidrossanitário, e as normas e documentos técnicos disponíveis exclusivamente no âmbito nacional.

\section{REVISÃO BIBLIOGRÁFICA}

\subsection{Programa de manutenção}

A durabilidade é a capacidade de desempenho ideal da estrutura ou de seus componentes em assegurar os requisitos de desempenho (POSSAN; DEMOLINER, 2013). Para uma longa durabilidade é necessário que seja realizada a inspeção e a manutenção do edifício, ou seja, o conceito de manutenção está intrínseco aos de durabilidade e vida útil de uma edificação (CREMONINI, 1988).

Para tanto, o programa de manutenção é a diretriz para implantação e execução da manutenção (CBIC, 2014). O programa de manutenção deve ser elaborado por profissional da área, em que deve constar a frequência para realizar a manutenção e inspeção de cada sistema que compõe a edificação, as informações a respeito da forma que deve ser feita a inspeção/manutenção, o responsável pela execução da manutenção, os documentos de referência e os recursos necessários. Devido ao planejamento desse tipo de programa tende a diminuir gastos com a manutenção predial (FERREIRA, 2017). Em caso de troca de síndico, o programa de manutenção torna-se essencial, pois relata todas as atividades agendadas que foram concluídas e aquelas que precisam ser realizadas 
(CREA-DF, 2014). Além disso, deve ser atualizado frequentemente para manter a integridade da manutenção proposta, sendo de grande importância a elaboração de relatórios das inspeções e manutenções realizadas para controlar o desempenho da manutenção, comparando as metas previstas e efetivas (ABNT, 2012). Devido ao volume de informações, a utilização de um software de manutenção torna-se viável, pois facilita o gerenciamento do programa de manutenção, tem uma menor porcentagem de erro e propõe diversos tipos de manutenção simultaneamente (ROSCOFF, 2019).

O Guia da CBIC instrui a elaboração do manual, oferecendo informações como responsabilidades técnicas, cuidados de uso, manutenção, perda de garantia de sistemas, atualização do manual entre outras orientações para elaboração e aplicação dos manuais de uso e áreas comuns. No que diz respeito às periodicidades de manutenção, são sugestões para as frequências de manutenção preventiva, contudo sem indicar a procedência técnica (CBIC, 2014). Devido à carência em informações relacionadas à periodicidade, o Conselho Regional de Engenharia e Agronomia (CREA) foi responsável por elaborar cartilhas e manuais para o auxílio da formulação de manuais do proprietário e das áreas comuns (CREA-DF, 2014).

Segundo a NBR 14037 (ABNT, 2014), no momento da entrega de um imóvel devem ser fornecidos o manual do proprietário e ao síndico o manual das áreas comuns. Ainda, é formulado o Termo de Garantia Definitivo, onde constarão os prazos de garantia a partir do Auto de Conclusão do imóvel, considerando os materiais e os sistemas construtivos. A construtora e/ou incorporadora deve fornecer modelo de programa de manutenção do edifício (CBIC, 2014).

O proprietário, por sua vez, precisa realizar a manutenção proposta pela construtora/incorporadora de acordo com o previsto, bem como o condomínio, na figura do síndico, têm responsabilidades na manutenção segundo a NBR 5674 (ABNT, 2012). Caso a manutenção não seja realizada em consonância com o proposto, poderá incorrer na perda da garantia do imóvel ou do equipamento (CBIC, 2014).

\section{MÉTODO DE PESQUISA}

\subsection{Escolha dos sistemas}

Existe uma grande quantidade de sistemas que necessitam de manutenção predial em um edifício, entre todos foram escolhidos os sistemas elétrico e hidrossanitário devido à quantidade de normas técnicas e informações disponíveis, pelo elevado número de manutenções propostas pelo Guia da CBIC (CBIC, 2014) e pela relevância frente aos usuários e ao desempenho.

\subsection{Revisão documental}

A revisão documental foi realizada com base em dados que possam apresentar periodicidades para a manutenção do sistema elétrico e hidrossanitário. Foi realizada busca online na bibliografia disponível no site do Sindicato da Indústria da Construção (Sinduscon) e do CREA dos estados brasileiros. Nessa busca foram encontradas duas cartilhas: no Distrito Federal e em Salvador, as quais apresentam dados sobre periodicidade, porém sem comprovação técnica do embasamento. Por fim, foi realizada pesquisa nas indústrias do mercado elétrico e hidrossanitário e catalogadas as normas da Associação Brasileira de Normas Técnicas (ABNT) ligadas aos sistemas em questão.

\subsubsection{Sistema elétrico}

A busca no mercado brasileiro foi realizada no site da Associação Brasileira da Indústria Elétrica e Eletrônica (ABINEE), órgão que possui associados de todos estados que atuam na fabricação de equipamentos para automação industrial, informática, telecomunicação 
entre outras ligadas à indústria elétrica e eletrônica. Neste caso, foram submetidos à revisão documental as empresas produtoras de materiais elétricos de instalação, pois esses materiais são citados no Guia da CBIC. Os associados informados no site da Abinee somaram um total de 55 empresas. O site de cada empresa foi verificado em busca de informações sobre a periodicidade da manutenção predial nos catálogos, certificados, manuais de uso e manuais técnicos dos produtos apresentados.

A revisão documental realizada nas normas técnicas ocorreu por meio da coleção virtual disponível no site da ABNT, em que todas as normas foram acessadas de forma online e na íntegra. Foram analisadas 23 normas do sistema elétrico (NBR 5361, NBR 5410, NBR 5418, NBR 5597, NBR 5598, NBR 5624, NBR 5674, NBR 7285, NBR 7286, NBR 7287, NBR 7288, NBR 8661, NBR 9326, NBR 6150, NBR 6527, NBR 13248, NBR 13300, NBR 13534, NBR 13570, NBR 13249, NBR 14039, NBR 14136, NBR 14306), iniciando pela instrução normativa utilizada pelo CBIC e seguindo a análise nas normas relacionadas com a NBR 5410 (ABNT, 2004). As normas passaram por uma análise, em que foram procuradas as palavras: periodicidade, manutenção e manutenção predial.

\subsubsection{Sistema hidrossanitário}

A revisão documental realizada para o sistema hidrossanitário foi efetuada da mesma forma que o sistema elétrico, de modo virtual por meio do site da Associação Brasileira dos Fabricantes de Materiais para Saneamento (ASFAMAS), entidade que possui 32 fabricantes associados de todo o Brasil. Foi realizada uma pesquisa no site de todos associados, buscando nos produtos informações sobre a periodicidade da manutenção predial por intermédio de documentos técnicos dos produtos, manuais de instalação, manuais de uso e certificado dos produtos.

A partir do catálogo da ABNT, foram analisadas, primeiro, as normas informadas no Guia da CBIC, em seguida, as normas recomendadas para a aplicação da NBR 5626 (ABNT, 1998). Foram analisadas 25 normas desse sistema (NBR 5626, NBR 5648, NBR 6493, NBR 7198, NBR 8160, NBR 8220, NBR 9077, NBR 10281, NBR 11304, NBR 13194, NBR 13713, NBR 13714, NBR 14122, NBR 14878, NBR 15206, NBR 15575-6, NBR 15705, NBR 15857, NBR 15884-1, NBR 15884-2, NBR 15884-3, NBR 15939, NBR 16749, NBR 17240), por meio das palavras-chave periodicidade, manutenção e manutenção predial.

\subsection{Tabulação de dados}

Todos os dados foram tabulados para posteriormente ocorreu a comparação com a periodicidade informada no Guia da CBIC, visando encontrar convergência entre os dados que pudessem comprovar a fonte técnica dos dados informados no Guia Nacional para a Elaboração do Manual de Uso, Operação e Manutenção das Edificações (CBIC, 2014). A tabulação foi realizada indicando as seguintes informações: subsistema (elemento) que necessita da inspeção/manutenção, ação necessária de manutenção (M), de inspeção (I) ou quando necessário $(\mathrm{N})$, atividade que deve ser realizada detalhada quando informada, periodicidade da manutenção ou inspeção, responsável técnico ou local e a fonte da informação. Os dados foram agrupados pelo seu subsistema (elemento) abrangendo todas as fontes, para facilitar a compreensão na comparação dos dados.

\subsection{Análise de dados}

Após a tabulação, foi realizada uma análise comparativa a fim de comparar as atividades, periodicidades, ações e responsáveis entre todas as fontes, conferindo a existência de semelhança entre as informações coletadas. Os dados encontrados nas cartilhas do CREA foram dispensados da análise devido à falta de comprovação técnica e a falta de informações concretas. A pesquisa nos sites dos Sinduscons resultou em dois guias 
encontrados: um manual do proprietário desenvolvido pelo Sinduscon de São Paulo, porém com informações idênticas ao da CBIC e um manual de garantias criado pelo estado do Pará, que informa sobre a necessidade da inspeção anual nos dois sistemas e qual o subsistema deve ser inspecionado, todavia apresenta carência de informações, impossibilitando a comparação com todos os outros dados. Importante ressaltar que a maioria das páginas virtuais do Sinduscon informa o link ou possibilita o download do Guia da CBIC (CBIC, 2014), devido ao trabalho em conjunto entre as partes.

\section{RESULTADOS}

Foram encontradas informações a respeito da periodicidade apenas nas normas NBR 5674 e NBR 13534, sendo essa relacionada aos estabelecimentos de saúde e elencando algumas periodicidades para inspeção e manutenção visando a segurança dos usuários. As normas descrevem, tanto para o sistema elétrico, quanto para o hidrossanitário, sobre a necessidade da inspeção visual no recebimento dos equipamentos, mas não informam periodicidades para manutenção durante o uso, apenas destacam que a periodicidade deve ser adequada a cada tipo de instalação (ABNT, 2004).

A pesquisa entre os fornecedores do sistema elétrico não resultou em informações capazes de comparar com os dados do Guia da CBIC, pois não foram encontrados dados a respeito da periodicidade da manutenção predial no site das empresas. Entretanto, para o sistema hidrossanitário foram encontradas orientações para instalação das bombas e possíveis causas e problemas, mas sobre a periodicidade para realização da manutenção predial foram encontradas informações apenas para os reservatórios. Nesse caso, consta orientação ao usuário para limpar os reservatórios com sabão neutro a cada seis meses, porém este tipo de manutenção é voltado para o cuidado com a água e não demonstra correlação com o desempenho do reservatório, sendo a informação descartada.

O Guia da CBIC (2014) possui diversas informações a respeito da manutenção do sistema elétrico e hidrossanitário, porém foram utilizadas apenas as informações semelhantes àquelas encontradas em normas para efeito comparativo, haja visto que para as outras informações disponíveis no Guia da CBIC não constaram parâmetros comparativos. Foram analisadas todas as instruções normativas utilizadas para criação do Guia da CBIC, além de normas específicas para acessórios dos sistemas elétrico e hidrossanitário, porém foram encontradas informações sobre periodicidade somente na NBR 5674 (ABNT 2012). Os dados apresentados no Quadro 1 estão relacionados às Instalações Elétricas.

Quadro 1 - Periodicidade indicada para o sistema de instalações elétricas

\begin{tabular}{|l|l|l|l|l|l|}
\hline $\begin{array}{l}\text { Subsistema } \\
\text { (elemento) }\end{array}$ & Ação & Atividade & $\begin{array}{l}\text { Periodici- } \\
\text { dade }\end{array}$ & Responsável & Fonte \\
\hline $\begin{array}{l}\text { Quadro de } \\
\text { distribuição }\end{array}$ & M & Reapertar todas as conexões & $\begin{array}{l}\text { Empresa } \\
\text { capacitada / } \\
\text { Empresa } \\
\text { especializada / } \\
\text { Equipe local }\end{array}$ & NBR 5674 \\
\cline { 2 - 6 } & $\mathrm{I} / \mathrm{M}$ & $\begin{array}{l}\text { Verificar e, se necessário, reapertar } \\
\text { as conexões do quadro de } \\
\text { distribuição }\end{array}$ & Anual & $\begin{array}{l}\text { Empresa } \\
\text { especializada }\end{array}$ & CBIC \\
\hline $\begin{array}{l}\text { Tomadas, } \\
\text { interruptore } \\
\text { s, pontos de } \\
\text { luz e cabos } \\
\text { de rede }\end{array}$ & $\mathrm{M}$ & $\begin{array}{l}\text { Verificar as conexões, estado dos } \\
\text { contatos elétricos e seus } \\
\text { componentes, e reconstituir onde } \\
\text { necessário }\end{array}$ & 2 anos & $\begin{array}{l}\text { Empresa } \\
\text { capacitada / } \\
\text { Empresa } \\
\text { especializada / } \\
\text { Equipe local }\end{array}$ & NBR 5674 \\
\cline { 2 - 6 } & I/M & $\begin{array}{l}\text { Verificar o estado dos contatos } \\
\text { elétricos. Caso possua desgaste, } \\
\text { substitua as peças (tomadas, } \\
\text { interruptores, pontos de luz e outros) }\end{array}$ & Anual & $\begin{array}{l}\text { Empresa } \\
\text { especializada }\end{array}$ & CBIC \\
\hline
\end{tabular}

Fonte: elaborado pelo autor 
Sobre o sistema elétrico, o subsistema quadro de distribuição de circuitos não apresentou informações concordantes, sendo orientado ao profissional realizar manutenção anual de reapertando das conexões. Já no caso das tomadas, interruptores e pontos de luz não houve semelhança na periodicidade informada e no responsável pela manutenção ou inspeção necessária.

Os dados apresentados no Quadro 2 dizem respeito às Instalações hidrossanitárias, em particular o sistema de água potável e não potável.

Quadro 2 - Periodicidade indicada para o sistema de instalações hidrossanitários

\begin{tabular}{|c|c|c|c|c|c|}
\hline $\begin{array}{l}\text { Subsistema } \\
\text { (elemento) }\end{array}$ & Ação & Atividade & $\begin{array}{l}\text { Periodici- } \\
\text { dade }\end{array}$ & Responsável & Fonte \\
\hline \multirow{2}{*}{$\begin{array}{l}\text { Água } \\
\text { Potável } \\
\text { (Bombas de } \\
\text { água } \\
\text { potável, } \\
\text { água } \\
\text { servida e } \\
\text { piscinas) }\end{array}$} & M & $\begin{array}{l}\text { Efetuar manutenção nas bombas de } \\
\text { recalque de água potável }\end{array}$ & Semestral & $\begin{array}{l}\text { Empresa } \\
\text { especializada }\end{array}$ & CBIC \\
\hline & I & $\begin{array}{l}\text { Verificar o funcionamento e alterna a } \\
\text { chave no painel elétrico para utilizá- } \\
\text { las em sistema de rodízio quando } \\
\text { aplicável }\end{array}$ & Quinzenal & Equipe Local & NBR 5674 \\
\hline \multirow{2}{*}{$\begin{array}{l}\text { Água } \\
\text { Potável } \\
\text { (Reservatór } \\
\text { ios, } \\
\text { torneiras de } \\
\text { boia e } \\
\text { chave de } \\
\text { boia) }\end{array}$} & I & $\begin{array}{l}\text { Verificar o nível dos reservatórios, o } \\
\text { funcionamento das torneiras de boia e } \\
\text { a chave de boia para controle de nível }\end{array}$ & Semanal & Equipe Local & $\mathrm{CBIC}$ \\
\hline & I & $\begin{array}{l}\text { Verificar o nível dos reservatórios e o } \\
\text { funcionamento das boias }\end{array}$ & Semanal & Equipe Local & NBR 5674 \\
\hline \multirow{2}{*}{$\begin{array}{l}\text { Água } \\
\text { Potável } \\
\text { (tubulações } \\
\text { ) }\end{array}$} & I & $\begin{array}{l}\text { Verificar as tubulações de água } \\
\text { potável para detectar obstruções, } \\
\text { perda de estanqueidade e sua fixação, } \\
\text { recuperar sua integridade onde } \\
\text { necessário }\end{array}$ & Anual & $\begin{array}{l}\text { Equipe local / } \\
\text { Empresa } \\
\text { capacitada }\end{array}$ & CBIC \\
\hline & $\mathrm{I} / \mathrm{M}$ & $\begin{array}{l}\text { Verificar as tubulações de água } \\
\text { potável e servida, para detectar } \\
\text { obstruções falhas ou entupimentos, e } \\
\text { fixação e reconstituir a sua } \\
\text { integridade, onde necessário } \\
\end{array}$ & Anual & $\begin{array}{l}\text { Empresa } \\
\text { especializada / } \\
\text { Equipe Local }\end{array}$ & NBR 5674 \\
\hline \multirow{2}{*}{\begin{tabular}{|l|} 
Água \\
Potável \\
(Metais \\
acessórios e \\
registros) \\
\end{tabular}} & I & $\begin{array}{l}\text { Verifique a estanqueidade dos } \\
\text { registros de gaveta }\end{array}$ & Semestral & Equipe Local & CBIC \\
\hline & $\mathrm{I} / \mathrm{M}$ & $\begin{array}{l}\text { Verificar os elementos de vedação dos } \\
\text { metais, acessórios e registros }\end{array}$ & Anual & Equipe Local & NBR 5674 \\
\hline \multirow{3}{*}{$\begin{array}{l}\text { Ralos, } \\
\text { grelhas, } \\
\text { calhas, } \\
\text { canaletas, } \\
\text { Caixas de } \\
\text { esgoto, de } \\
\text { gordura e } \\
\text { de águas } \\
\text { servidas } \\
\end{array}$} & M & $\begin{array}{l}\text { Limpar o sistema das águas pluviais e } \\
\text { ajustar a periodicidade em função da } \\
\text { sazonalidade, especialmente em } \\
\text { épocas de chuvas intensas } \\
\end{array}$ & Mensal & Equipe Local & NBR 5674 \\
\hline & M & $\begin{array}{l}\text { Limpar e regular os sistemas de } \\
\text { queimadores e filtros de água, } \\
\text { conforme instruções dos fabricantes }\end{array}$ & 2 meses & $\begin{array}{l}\text { Empresa } \\
\text { Capacitada }\end{array}$ & CBIC \\
\hline & M & Efetuar limpeza geral & 3 meses & Equipe Local & NBR 5674 \\
\hline
\end{tabular}

Fonte: elaborado pelo autor

Em relação ao sistema hidrossanitário, no caso do subsistema bombas de água potável não houve semelhança em nenhuma informação encontrada na NBR 5674 com o Guia da CBIC. Já as informações a respeito dos reservatórios convergiram entre a normas e o Guia da CBIC, instruindo a equipe local a verificar o nível dos reservatórios e o funcionamento das boias semanalmente. Para o elemento tubulações foi identificada convergência de 
informações entre a norma e o Guia da CBIC, instruindo o usuário a verificar as tubulações e reconstituir a sua integridade, onde necessário, com uma periodicidade anual. Para os metais, acessórios e registros não foi encontrada analogia entre os dados, sendo informado a necessidade de inspeção semestral pelo Guia da CBIC e inspeção/manutenção anual pela NBR 5674. Acerca dos filtros como caixa de esgoto e gordura, as informações não foram compatíveis: periodicidade para manutenção de 2 meses no Guia da CBIC e 3 meses na NBR 5674 para a atividade de limpeza dos filtros.

\section{CONCLUSÃO}

A manutenção predial preventiva tem extrema importância para a conservação do edifício e sua prática pode evitar danos e gastos com manutenções corretivas. Por outro lado, o excesso de manutenção e a confecção de um manual com periodicidades indevidas pode também gerar gastos desnecessários prejudicando os usuários.

O comparativo entre os dados informados no Guia da CBIC com aqueles de fabricantes, das normas pertinentes, das cartilhas e dos documentos do CREA e Sinduscon não convergiu. Observaram-se diversas divergências, tanto nas atividades, quanto na periodicidade, sendo que o Guia da CBIC apresentou informações a respeito da periodicidade dos sistemas selecionados e para outros 38 subsistemas, o que não consta em nenhuma das normas da ABNT.

Disso, infere-se que existe uma grande falta de informações sobre a manutenção predial. Por parte dos fornecedores, percebe-se que a maioria dos produtos consultados, tanto do sistema elétrico, quanto hidráulico, não é informado nenhum tipo de manutenção para manter o desempenho e funcionamento, dificultando a especificação necessária realizada pelo projetista. As poucas informações, a respeito da manutenção predial e de sua periodicidade, dificultam a verticalização da pesquisa sobre o assunto, refletindo em um comparativo com número sucinto de dados. Mesmo assim, a tabela comparativa demonstrou que as informações contidas no Guia da CBIC não são similares às informações técnicas coletadas e que nem mesmo a base normativa informada esclarece as fontes técnicas oriundas das atividades e periodicidades propostas.

Quanto às limitações do trabalho, o Guia da CBIC apresentou os cuidados de uso, manutenção e perda de garantia para 52 sistemas, entretanto o enfoque da pesquisa foi apenas o sistema elétrico e hidrossanitário. Ainda, cabe destacar que todas as informações obtidas em normas e com fornecedores são de origem exclusivamente nacional.

A contribuição central do artigo diz respeito às periodicidades e às atividades de manutenção preventiva propostas pelo Guia da CBIC, especificamente para o sistema hidrossanitário e elétrico, em que não foi identificada fundamentação técnica. A partir dos resultados alcançados, sugere-se para trabalhos futuros os seguintes estudos: (a) influência da variação climática na periodicidade da manutenção; e (b) orçamentação da manutenção para um edifício residencial utilizando os parâmetros do Guia da CBIC.

\section{REFERÊNCIAS}

ABNT. ASSOCIAÇÃO BRASILEIRA DE NORMAS TÉCNICAS. NBR 5410: Instalações elétricas de baixa tensão. Rio de Janeiro, 2004.

NBR 5626: Instalação predial de água fria. Rio de Janeiro, 1998.

NBR 5674: Manutenção de edificações - requisitos para o sistema de gestão de manutenção. Rio de Janeiro, 2012.

NBR 13534: Instalações elétricas de baixa tensão - Requisitos específicos para instalação em estabelecimentos assistenciais de saúde. Rio de Janeiro, 2008. 
. NBR 14037: Diretrizes para elaboração de manuais de uso, operação e manutenção das edificações - Requisitos para elaboração e apresentação dos conteúdos. Rio de Janeiro, 2014.

NBR 15575-1: Edificações habitacionais - Desempenho: requisitos gerais. Rio de Janeiro, 2013.

NBR 16280: Reforma em edificações - Sistema de gestão de reformas - Requisitos. Rio de Janeiro, 2015.

BORTOLINI, R.; FORCADA, N. Building inspection system for evaluating the technical performance of existing buildings. Journal of Performance of Constructed Facilities, v. 32, n. 5, p. 04018073, 2018.

CBIC - CÂMARA BRASILEIRA DA INDÚSTRIA DA CONSTRUÇÃO. Guia nacional para a elaboração do manual de uso, operação e manutenção das edificações. Fortaleza: Gadioli Cipolla Branding e Comunicação, 2014, 185p.

. Economia em perspectiva. Brasília, DF, 2018. Disponível em: https://cbic.org.br/informativo/.

CREA-DF - CONSELHO REGIONAL DE ENGENHARIA E AGRONOMIA DO DISTRITO FEDERAL. Cartilha do síndico obras e serviços de engenharia e de agronomia: o que é preciso saber. Distrito Federal, 2014, 44p.

CREMONINI, R. A. Incidência de manifestações patológicas em unidades escolares na região de Porto Alegre: recomendações para projeto, execução e manutenção. 1988. 153f. Dissertação (Mestrado em Engenharia) - Universidade Federal do Rio Grande do Sul, Porto Alegre.

DAS, S.; CHEW, M. Y. L. Generic method of grading building defects using FMECA to improve maintainability decisions. Journal of Performance of Constructed Facilities, v. 25 , n. 6, p. 522-533, 2011.

FERREIRA, F. M. C. Modelo para gestão de manutenção predial em universidades públicas: caso das IFES mineiras. 2017. 187f. Tese (Doutorado em Engenharia Civil) Universidade Federal de Ouro Preto, Ouro Preto.

MAURÍCIO, F. M. M. P. Aplicação de ferramentas de facility management à manutenção técnica de edifícios de serviços. 2011. 159f. Dissertação (Mestrado em Engenharia Civil) - Universidade Técnica de Lisboa, Portugal.

POSSAN, E.; DEMOLINER, C. A. Desempenho, durabilidade e vida útil das edificações: abordagem geral. Revista Técnico Científica, Conselho Regional de Engenharia e Agronomia do Paraná (CREA-PR), n. 1, p. 1-14, 2013.

ROSCOFF, N. S. Desenvolvimento de um software para a gestão da manutenção predial preventiva. 2019. 142f. Dissertação (Mestrado em Tecnologia e Gestão da Inovação) Universidade Comunitária da região de Chapecó, Chapecó.

VIEIRA, F. N. Proposta de elaboração de plano de manutenção para edificações a partir da obrigatoriedade legal da inspeção predial no contexto urbano das cidades. Dissertação (Mestrado em Engenharia Urbana) - Universidade Federal do Rio de Janeiro, Rio de Janeiro, 2015. 